\title{
Pronóstico Numérico del Viento a Mesoescala y a Corto Plazo para el Aeropuerto Internacional "José Martí” de La Habana
}

\author{
Arlett Díaz-Zurita $^{1}$ (D), Carlos Manuel Góngora-González ${ }^{1}$, Alexander Lobaina-LaÓ ${ }^{1}$, \\ Albenis Pérez-Alarcón ${ }^{2,3}$ (D), Patricia Coll-Hidalgo ${ }^{2}$ \\ ${ }^{1}$ Empresa Cubana de Navegación Aérea, Carretera Panamericana y Final, Boyeros, La Habana, \\ Cuba. \\ ${ }^{2}$ Departamento de Meteorología, Instituto Superior de Tecnologías y Ciencias Aplicadas, \\ Universidad de La Habana, La Habana, Cuba. \\ ${ }^{3}$ Environmental Physics Laboratory, CIM-UVigo, Universidad de Vigo, Ourense, España.
}

Recebido em: 29 de Outubro de 2019 - Aceito em: 26 de Agosto de 2020

\section{Resumen}

En el Aeropuerto Internacional "José Martí" de La Habana (MUHA), el nivel de exactitud que presentan los pronósticos de dirección del viento se aproximan a un 70\%, los mismos no cumplen con los requisitos establecidos por las regulaciones aeronáuticas que exigen que la efectividad sea mayor al $85 \%$. Por ello, se implementó un pronóstico numérico del campo de viento a mesoescala y a corto plazo utilizando los modelos WRF-NMM y Masa Consistente, que pretende mejorar el pronóstico realizado por el Ogimet, herramienta numérica de pronóstico del viento empleada en el MUHA. Se experimentó con distintos métodos de interpolación, obteniendo los mejores resultados con el vecino natural con la corrección del modelo de Masa Consistente. Se desarrolló una herramienta computacional capaz de ofrecer los pronósticos numéricos de campo de viento para el MUHA correspondientes a las 0000, 0600, 1200, 1800 UTC, con una habilidad superior al Ogimet y con una efectividad del 91\% para el pronóstico numérico del campo de viento.

Palabras-clave pronóstico, viento, mesoescala, masa consistente, WRF-NMM.

\section{Numerical Wind Forecast at Mesoscale and Short Term for José Martí International Airport in Havana}

\begin{abstract}
At the "José Martí" International Airport in Havana (MUHA), the level of accuracy presented by wind direction forecasts is close to $70 \%$, they do not meet the requirements established by the aeronautical regulations which effectiveness must be higher than $85 \%$. Therefore, a numerical forecast of the wind field was implemented at mesoscale, in a short term, using the WRF-NMM and Consistent Mass models that are aimed to improve the forecast made by the Ogimet, a wind forecast numerical tool used in the MUHA. It was experimented with different interpolation methods; the best results were obtained on the natural neighbor with the correction of Consistent Mass model. A computational tool was developed which provided the numerical wind field forecasts for the MUHA corresponding to the 0000, 0600, 1200 and 1800 UTC which have a greater ability than the Ogimet and with an effectivity of $91 \%$ for numerical forecast of the wind field.
\end{abstract}

Keywords: forecast, wind, mesoscale, consistent mass, WRF-NMM.

\section{Introducción}

El factor meteorológico más importante para el correcto desempeño de la aviación es el viento, los cambios en su velocidad afectan a la aeronave en vuelo, debido que el flujo del aire alrededor de las alas es el origen de su sustentación (De Armas et al., 2019). Los momentos más críticos en los vuelos de las aeronaves son las operaciones de aterrizaje y despegue, donde es más proclive la ocurrencia de accidentes; por ello, resulta indispensable la realización

Autor por correspondencia: Arlett Díaz-Zurita, e-mail: adzurita378@gmail.com. 
de observaciones meteorológicas y de estudios climáticos que sirvan de caracterización de las variables meteorológicas en el aeropuerto, para la elaboración de los pronósticos de aeródromos (IACC, 2017).

El pronóstico local de la dirección y fuerza del viento es una tarea compleja debido a que el viento tiene una alta variabilidad en cortos períodos de tiempo, depende de la orografía de la localidad y sobre él actúan numerosas fuerzas, que suelen variar su dirección y velocidad. Dada la imposibilidad de determinar el valor de la dirección y velocidad del viento en cada punto del espacio y su evolución temporal utilizando instrumentos convencionales se recurre a la simulación de la dinámica del fluido, siendo la modelación una de las técnicas más utilizadas (Jiménez, 2012)

Los Modelos Matemáticos de campos de viento se pueden clasificar en Modelos de Pronóstico o Dinámicos y Modelos de Diagnóstico o Cinemáticos. Los Modelos de Pronóstico se basan en la solución de ecuaciones hidrodinámicas y termodinámicas que dependen del tiempo, modificadas para su aplicación a la atmósfera. Los Modelos de Diagnóstico eliminan directamente de sus ecuaciones la dependencia del tiempo y si la principal restricción que se le impone es la conservación de la masa, se denominan modelos de Masa Consistente (MMC) (Ratto et al., 1994).

En la bibliografía consultada relacionada con la aviación se destacan los estudios de Robles (2003) y Stolz et al. (2012). Robles (2003) resaltó que, aunque la cizalladura del viento está presente en toda la tropósfera, la que puede considerarse significativa es la que acontece desde el suelo hasta unos 1500 pies (ft), con una razón de 10 nudos (kt) por cada $100 \mathrm{ft}$. Stolz et al. (2012) definieron los horarios de mínima y máxima velocidad del viento, así como la dirección del viento predominante en el Aeropuerto Internacional Tobías Bolaños Palma de Costa Rica.

En el caso de Cuba, particularmente en el MUHA, los estudios caracterizan los fenómenos extremos para la aviación; entre ellos se destacan: Pérez (2006), Entenza (2009) y Torres (2011). Todos ellos corroboran que entre los fenómenos más peligrosos para la aviación se encuentran las tormentas eléctricas, las fuertes precipitaciones, los vientos cruzados en superficie, la niebla, la neblina y la turbulencia mecánica.

Relacionados con la modelación de campos de vientos, en el ámbito internacional, se destacan los estudios realizados por: Montero et al. (2005), Rodríguez et al. (2009), Jácome (2014) y Méndez y Calvo (2016). Montero et al. (2005) implementaron un modelo tridimensional de Masa Consistente para realizar la simulación numérica de campos de vientos en la isla de Gran Canaria. Rodríguez et al. (2009) presentaron un modelo tridimensional de Masa Consistente para realizar ajustes de campos de viento utilizando elementos finitos con mallas adaptadas de tetraedro, con el objetivo de obtener una mejora de la pre- dicción del viento a escala local en la isla de Gran Canaria. Jácome (2014) utilizó un MMC para generar campos de viento $3 \mathrm{D}$, a partir de datos experimentales interpolados, en un dominio con paredes artificiales para dos casos de condiciones de frontera: (1) Dirichlet sobre las paredes artificiales y (2) Neumann en las paredes verticales y Dirichlet en la tapa. Los resultados numéricos mostraron que se obtiene una mejor aproximación cuando se imponen las condiciones de frontera del caso (2). Méndez y Calvo (2016) realizaron una evaluación de un modelo 3D de elementos finitos y un MMC para la predicción de viento a muy alta resolución. Los mismos concluyeron que el modelo de elementos finitos permite utilizar mallas irregulares que se adaptan a orografías complejas como la de la isla de Gran Canaria, pudiendo realizar estimaciones de viento a resoluciones de unas decenas de metros y que el MMC mejora las predicciones de viento en promedio, pero tiene dificultades para simular los valores extremos.

En el caso de Cuba, se destaca la investigación llevada a cabo por Jiménez (2012), quien implementó un modelo bidimensional de Masa Consistente capaz de simular campos diagnósticos de velocidad del viento en zonas de orografía compleja en Guanito, Pinar del Rí o, Cuba.

Una gran variedad de estudios vinculados con el viento se han desarrollado en el aeródromo objeto de esta investigación, los mismos han sido mayoritariamente estadístico-descriptivos y climatológicos, por lo que hasta el momento no se ha llevado a cabo una investigación en la que se realice un pronóstico numérico del campo de viento a mesoescala y a corto plazo para el Aeropuerto Internacional "José Martî" de La Habana, lo cual es fundamental para incrementar la exactitud de los pronósticos del campo de viento. Por ello se propone como objetivo del presente trabajo:

Desarrollar el pronóstico numérico del campo de viento a mesoescala y a corto plazo para el Aeropuerto Internacional "José Martí" de La Habana utilizando los modelos WRF-NMM y Masa Consistente cumpliendo con la Regulación Aeronáutica Cubana.

\section{Materiales y Métodos}

\subsection{Zona de estúdio}

Para la elaboración de esta investigación se seleccionó el Aeropuerto Internacional "José Martí" de La Habana. Esta instalación aeroportuaria se ubica al suroeste (SW) de la provincia de La Habana, a unos $13 \mathrm{~km}$ de la costa norte, en el municipio de Boyeros, en los $22.99^{\circ}$ de latitud Norte (N) y los $82.40^{\circ}$ de longitud Oeste (W). Posee una única pista de $4 \mathrm{~km}$ de longitud, orientada en dirección SW-Nordeste (NE), cuyas cabeceras están designadas de acuerdo a la orientación con que las aeronaves ejecutan la aproximación: Runway (RWY) $06\left(58^{\circ}\right)$ y RWY 24 (238 ) (IACC, 2014). 


\subsection{Herramientas y modelos empleados}

\subsubsection{Weather Research and Forecasting (WRF)}

El Weather Research and Forecasting (WRF) es un sistema numérico de mesoescala de nueva generación diseñado para servir a las necesidades de investigación y predicción operativa atmosférica. Está compuesto por dos núcleos dinámicos: el ARW (Advanced Research WRF) y el NMM (Nonhydrostatic Mesoescale Model), un sistema de asimilación de datos y una arquitectura de software que facilita su corrida en paralelo y la extensibilidad del sistema (Janjic et al., 2014). En la presente investigación se empleó el NMM en su versión 3.6.1.

El núcleo dinámico NMM es el programa dentro de la infraestructura del modelo que realiza los principales cálculos y produce la simulación. Es adecuado para su uso en una amplia gama de aplicaciones, en escalas que van desde metros hasta miles de kilómetros, que incluyen: NWP (Numerical Weather Prediction) en tiempo real, investigación de pronósticos y parametrizaciones, y aplicaciones de modelos acoplados (Janjic et al., 2014).

\subsubsection{Modelo de Masa Consistente}

Los MMC están basados en la ecuación de continuidad para un fluido incompresible (Eq. (1)), considerando la densidad del aire constante en todo el dominio $\Omega$ (Eq. (2)), con un campo de velocidades $\vec{u}(u, v, w)$ y condiciones de impenetrabilidad sobre el terreno $\Gamma_{b}$ y en la frontera superior (Eq. (3)) (Rodríguez et al., 2009):

$$
\begin{gathered}
\frac{\partial p}{\partial t}+\nabla \cdot(\rho \vec{u})=0 \\
\nabla \cdot \vec{u}=0 \text { en } \Omega \\
\overrightarrow{n \cdot u}=0 \text { en } \Gamma_{b}
\end{gathered}
$$

A partir de las condiciones (2) y (3), se formula un problema de mínimos cuadrados en el dominio $\Omega$ con el objetivo de ajustar $\vec{u}(u, v, w)$, a partir de las velocidades observadas $\overrightarrow{u_{0}}\left(u_{0}, v_{0}, w_{0}\right)$, de acuerdo con el funcional:

$$
\begin{aligned}
& E(u, v, w)=\iiint_{\Omega}\left[\alpha_{1}^{2}\left\{\left(u-u_{0}\right)^{2}+\left(v-v_{0}\right)^{2}\right\}\right. \\
& \left.+\alpha_{2}{ }^{2}\left(w-w_{0}\right)^{2}\right] d x d y d z
\end{aligned}
$$

donde $\vec{u}(u, v, w)$, son las componentes del viento calculadas por el modelo mediante ajuste, $\alpha_{1}$ y $\alpha_{2}$ son los módulos de precisión de Gauss (Montero et al., 2005, Rodríguez et al, 2009).

El campo buscado $\vec{v}(u, v, w)$ sería la solución del problema, tal que:

$$
\mathrm{E}(\vec{v})=\min E(\vec{u}), \quad \mathrm{K}=\left\{\vec{u} ; \nabla \cdot \vec{u}=0, \overrightarrow{n \cdot} \cdot \vec{u} \mid \Gamma_{b}=0\right\}
$$

Este problema es equivalente a encontrar el punto silla en $[\vec{u}, \phi]$ del Lagrangiano:

$$
L(\vec{u}, \lambda)=\mathrm{E}(\vec{u})+\int_{\Omega} \lambda \nabla \cdot \vec{u} d \Omega
$$

El campo de solución se obtiene a partir de las ecuaciones de Euler-Lagrange:

$$
\vec{v}=\overrightarrow{v_{0}}+\mathrm{T} \nabla \phi
$$

siendo $\phi$ el multiplicador de Lagrange y $T=\left[T_{h}, T_{h}, T_{v}\right]$ el tensor diagonal de transmisión:

Si $\alpha_{1}$ y $\alpha_{2}$ se consideran constantes en todo el dominio, la formulación variacional conduce a una ecuación elíptica definida en $\phi$ :

$$
-\nabla \cdot(\mathrm{T} \nabla \phi)=\nabla \cdot \overrightarrow{u_{0}}
$$

que se completa con la condición de Dirichlet (Eq. (9)) nula en las fronteras permeables (fronteras verticales del dominio) y la condición de Neumann (Eq. (10)) en las impermeables (terreno y en la frontera superior):

$$
\begin{gathered}
\phi=0 \text { en } \Gamma_{a} \\
\vec{n} \cdot \nabla \phi=-\overrightarrow{n \cdot v_{0}} \text { en } \Gamma_{b}
\end{gathered}
$$

Al considerar $T_{h}$ y $T_{v}$ constantes y eliminando la componente vertical (dos dimensiones) se obtiene:

$$
\frac{\partial^{2} \phi}{\partial x^{2}}+\frac{\partial^{2} \phi}{\partial y^{2}}=-\frac{1}{T_{h}}\left(\frac{\partial u_{0}}{\partial x}+\frac{\partial v_{0}}{\partial y}\right)
$$

donde: $T_{h}=\frac{1}{2 \alpha_{1}{ }^{2}}$

\subsubsection{Ogimet}

Ogimet es una herramienta numérica que utiliza mensajes meteorológicos de tipo alfanuméricos como los METAR (METeorological Aerodrome Report) y los SPECI (SPEcial Meteorological Reports), emplea datos del modelo de predicción GFS (Global Forecast System) de la NOAA (National Oceanic and Atmospheric Administration), con una resolución espacial de $0.5^{\circ}$. A estos datos, se le incluyen pequeñas correcciones en los datos de superficie para tener en cuenta el error en el modelo de la topografía respecto a los valores reales, fundamentalmente la altitud del suelo y la fracción de suelo seco en los alre- 
dedores (disponible en la página web del Ogimet). Esta herramienta proporciona salidas gráficas de las distintas variables meteorológicas en los distintos niveles verticales y actualmente es de la más empleada por los meteorólogos aeronáuticos del MUHA para realizar los pronósticos de campo de viento.

\subsection{Métodos de Interpolación utilizados}

\subsubsection{Interpolación Lineal}

Según Álvarez et al. (2009) la interpolación lineal utiliza un polinomio de interpolación de grado 1 , que se ajusta a los valores en los puntos $x_{1}$ y $x_{2}$ :

$$
y=\frac{x-x_{1}}{x_{2}-x_{1}}\left(y_{2}-y_{1}\right)+y_{1}
$$

\subsubsection{Spline cúbico}

Dada una función $f$ definida en $[a, b]$ y en un conjunto de nodos $a=x_{0}<x_{1}<\cdots<x_{n}=b$, el spline cúbico, $s$, para $f$, está representado por la siguiente expresión:

$$
\begin{aligned}
s(x) & =\frac{v^{3} M_{i}+u^{3} M_{i+1}}{6 h_{i}}+\frac{v y_{i}+u y_{i+1}}{h_{i}} \\
& -\frac{h_{i}\left(v M_{i}+u M_{i+1}\right)}{6}
\end{aligned}
$$

$$
\begin{aligned}
y_{i}= & \frac{1}{6} h_{i}{ }^{2} M_{i}+k_{1} x_{i}+k_{2} \quad y_{i+1}=\frac{1}{6} h_{i}{ }^{2} M_{i+1}+k_{1} x_{i+1}+k_{2} \\
& \ddot{s}\left(x_{i}\right)=M_{i} \quad \ddot{s}\left(x_{i+1}\right)=M_{i+1} \\
& u=x-x_{i} \quad v=x_{i+1}-x \\
& h_{i}=x_{i+1}-x_{i}
\end{aligned}
$$

donde $k_{1} y k_{2}$ son constantes, $h_{i}$ las longitudes de los tramos en que están definidos los $n$ polinomios del spline (Álvarez et al., 2009).

\subsubsection{Interpolación del vecino natural}

La interpolación del vecino natural se basa en la teselación Voronoi de un conjunto discreto de puntos espaciales, la misma proporciona una aproximación más suave a la función "verdadera" subyacente (Sibson, 1981). La ecuación básica en 2D es:

$$
G(x, y)=\sum_{i=1}^{n} w_{i} f\left(x_{i}, y_{i}\right)
$$

donde $w_{\mathrm{i}}$ son los pesos y $f\left(x_{i}, y_{i}\right)$ son los datos conocidos en $\left(x_{i}, y_{i}\right)$.

\subsubsection{Funciones de base radial (RBF)}

Las RBF son funciones que satisfacen que $\phi(r)=\Phi(|| x||)$, o de forma alternativa $\phi(r)=\Phi_{k}(x)=$ $\Phi\left(\left\|x-x_{k}\right\|\right)$ (Lazzaro y Montefusco, 2002):

$$
P_{f}=\sum_{k=1}^{n} c_{k} \Phi\left(\left\|x-x_{k}\right\|\right) \quad x \in R^{\delta}
$$

donde las constantes $c_{\mathrm{k}}$ son los pesos y $x_{k}$ la distancia a algún centro.

\subsubsection{Interpolación del vecino más cercano}

La interpolación del vecino más cercano se basa en la selección del valor del punto más cercano y no tiene en cuenta los valores de los puntos vecinos en absoluto, rindiendo un interpolador por tramos constante (Hernández y León, 2016).

\subsection{Estadígrafos empleados}

En los siguientes estadígrafos $x_{i}$ representa a los valores observados en el centro (MID) y las cabeceras (RWY 06 y RWY 24) de la pista del MUHA, $y_{i}$ los valores simulados en dichas posiciones, $x_{i}$ la media de los valores reales y $\bar{y}_{i}$ la media de los modelados. Fueron empleados según los definen Jolliffe y Stephenson (2003).

Sesgo (BIAS)

$$
B I A S=\frac{\sum_{i=1}^{N}\left(y_{i}-x_{i}\right)}{N}
$$

Error Absoluto Medio (MAE)

$$
M A E=\frac{\sum_{i=1}^{N}\left|y_{i}-x_{i}\right|}{N}
$$

Correlación de Pearson $\left(r_{p}\right)$

$$
r_{p}=\frac{\sum_{i=1}^{N}\left(x_{i}-\bar{x}_{i}\right)\left(y_{i}-\bar{y}_{i}\right)}{\sqrt{\sum_{i=1}^{N}\left(x_{i}-\bar{x}_{i}\right)^{2}\left(y_{i}-\bar{y}_{i}\right)^{2}}}
$$

Habilidad de Pronóstico (SS)

$$
\mathrm{SS}=1-\sqrt{\frac{\sum_{i=1}^{N}\left(y_{i}-x_{i}\right)^{2}}{\frac{1}{N} \sum_{i=1}^{N} x_{i}{ }^{2}}}
$$

Índice de Ajuste (IA)

$$
I A=1-\frac{\sum_{i=1}^{N}\left(x_{i}-y_{i}\right)^{2}}{\sum_{i=1}^{N}\left(\left|y_{i}-\bar{x}_{i}\right|+\left|x_{i}-\bar{x}_{i}\right|\right)^{2}}
$$

Índice de Dispersión (SI)

$$
S I=\frac{\sqrt{\frac{\sum_{i=1}^{N}\left(y_{i}-x_{i}\right)^{2}}{N}}}{\frac{1}{N} \sum_{i=1}^{N} x_{i}{ }^{2}}
$$


Error relativo $(\varepsilon)$

$$
\varepsilon(\%)=\frac{\left|y_{i}-x_{i}\right|}{x_{i}} * 100
$$

Para calcular la efectividad de los pronósticos se empleó la siguiente ecuación:

$$
\text { efectividad }(\%)=100-\varepsilon(\%)
$$

\subsection{Diseño de los experimentos}

El WRF-NMM fue inicializado a partir de las salidas pronóstico del modelo GFS. Los datos de inicialización fueron descargados del NCEP (National Center for Environmental Prediction). Los datos de viento de las salidas numéricas del modelo WRF-NMM que se emplearon para el desarrollo de esta investigación corresponden al período 2017-2019.

Para la obtención de los datos reales de viento, en el centro y las cabeceras de la pista del MUHA, se emplearon los registros meteorológicos del campo de viento de la estación semiautomática Väisälä de dicho aeródromo. Esta estación cuenta con tres anemómetros instalados a lo largo de la pista, a una distancia mínima normada ${ }^{1}$, para no ser considerados obstáculos para las operaciones aéreas (Fig. 1).
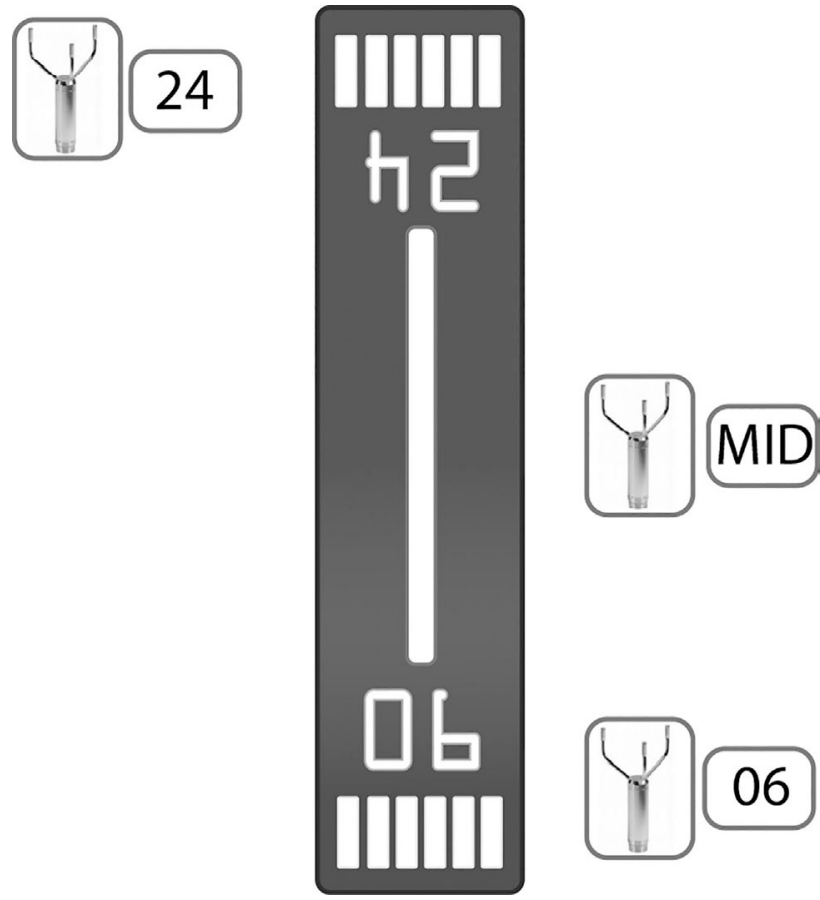

Figura 1 - Distribución de los sensores de viento en el campo del aeródromo del MUHA
El WRF-NMM fue inicializado a las 0000 y 1200 UTC, para la realización de las corridas fueron utilizadas resoluciones espaciales de 27, 9 y $3 \mathrm{~km}$, con actualización de las condiciones de frontera cada 6 horas y salidas numéricas en intervalos de tres horas. Para realizar este estudio se escogió el dominio de $3 \mathrm{~km}$ de resolución espacial (Fig. 2A). En el dominio de $3 \mathrm{~km}$ de resolución la densidad de los nodos en el área de interés (una vecindad de $8 \mathrm{~km}$ del MUHA) era baja y no había coincidencia de los mismos con la pista del aeródromo objeto de estudio (Fig. 2A). Por tales razones se desarrolló una nueva rejilla rectangular, cuyo centro se encuentra en el centro de la pista del MUHA, la misma tiene en cuenta la orientación y largo de la pista $\left(60^{\circ}\right.$ y $4 \mathrm{~km}$ respectivamente) y en ella se hacen coincidir 5 puntos: uno en el centro de la pista, uno en cada cabecera y los otros 2 puntos a $1 \mathrm{~km}$ del centro (Fig. 2B). Teniendo en cuenta todos estos aspectos la malla utilizada tiene una resolución longitudinal de $0.5 \mathrm{~km} \mathrm{y}$ latitudinal de $0.87 \mathrm{~km}$.
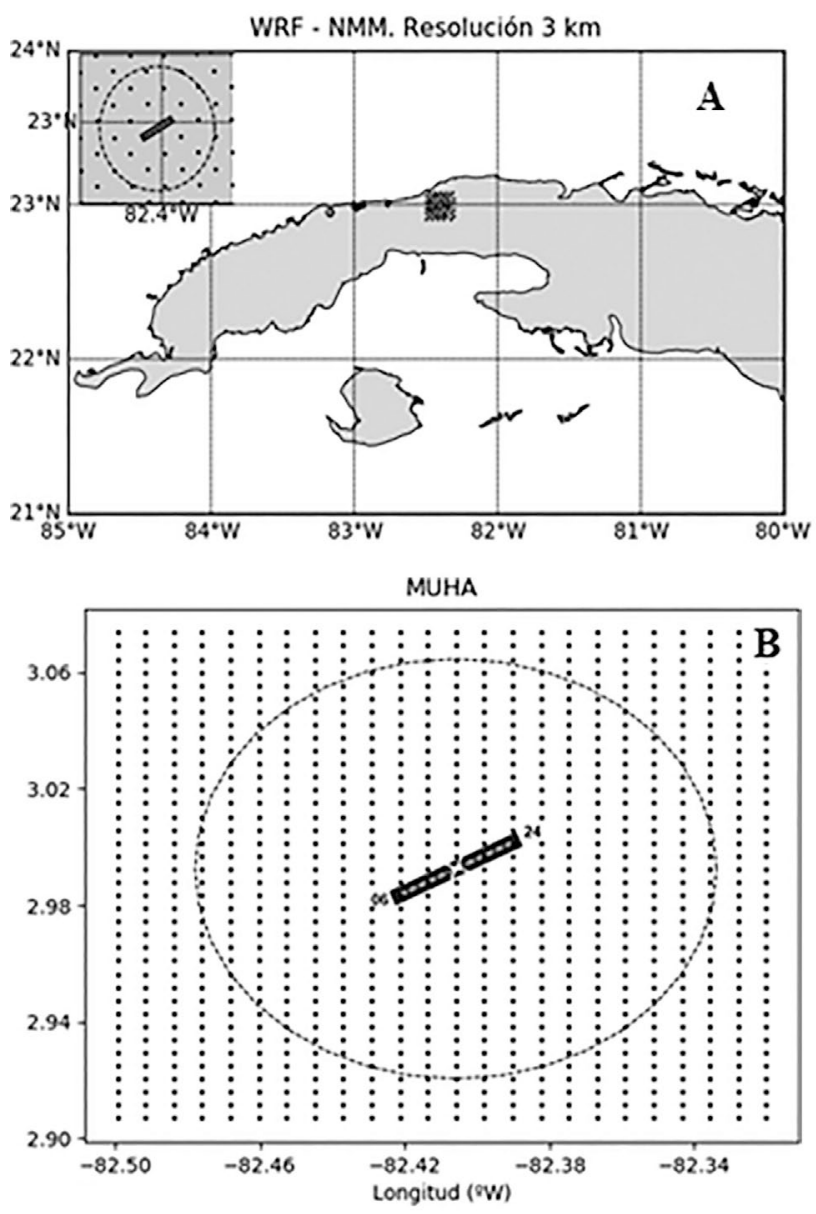

Figura 2 - A) Dominio utilizado para las corridas del modelo WRFNMM. B) Rejilla rectangular empleada para la interpolación.

${ }^{1}$ Distancia mínima normada: a $150 \mathrm{~m}$ de la zona de toma de contacto de las cabeceras y el centro de la pista. 
El campo de viento simulado por el modelo WRFNMM es interpolado espacialmente, mediante los métodos descritos en la sección 2.3, a la rejilla creada, para obtener la dirección y fuerza del viento en el centro y las cabeceras de la pista del MUHA; así como en el área de interés. El campo interpolado se corrige con el modelo Masa Consistente, se interpola temporalmente en intervalos de 1 hora mediante el spline cúbico y se realiza el pronóstico numérico del campo de viento para el MUHA, a partir de las $06,12,18$ y 24 horas de inicializado el modelo, estos pronósticos tienen un plazo de validez de 24 horas.

Las corridas del modelo WRF-NMM se realizaron conforme la Tabla 1.

Estas parametrizaciones fueron seleccionadas a partir de estudios de sensibilidad realizados en Cuba por Sierra et al., 2015 y Pérez Bello et al., 2019.

\section{Resultados y Discusión}

Se alcanzaron resultados similares con la inicialización de las 0000 y 1200 UTC, a continuación, se muestran los alcanzados con la corrida inicializada a las 0000 UTC.

\subsection{Análisis de la interpolación realizada}

En la Fig. 3, se puede apreciar como el campo de viento interpolado con el vecino más cercano no es capaz de representar bien el comportamiento de las isotacas del campo de viento del WRF-NMM, debido a que dicho método no tiene en cuenta la condición de que el campo sea no divergente; además, este método sobrestima el valor de la fuerza del viento en áreas cercanas al centro y a
Tabela 1 - Configuración utilizada en el modelo WRF-NMM.

\begin{tabular}{lc}
\hline Tipo de parametrizaciones & Parametrizaciones \\
\hline Microfísica & WSM5 (WRF Single-Moment 5-class) \\
Radiación de onda larga & RRTM (Rapid Radiative Transfer Model) \\
Radiación de onda corta & Dudhia \\
Capa superficial & Eta similarity \\
Modelo superficial & Noah Land Surface Model \\
Capa fronteriza planetaria & MYJ (Mellor-Yamada-Janjic) \\
Cúmulos & Kain-Fritsch \\
\hline
\end{tabular}

la RWY 06 aproximadamente en 2 kt. También se observa como al aplicarle a dicho método de interpolación una corrección con el modelo de Masa Consistente los resultados mejoran significativamente, tanto en dirección, como en velocidad del viento, siendo estos muy próximos a los valores estimados por el modelo WRF-NMM; ya que dicho método tiene en cuenta la conservación de la masa, la no divergencia del campo, las condiciones de impenetrabilidad sobre el terreno y la condición de Dirichlet nula en las fronteras permeables (fronteras verticales del dominio). Además, se puede observar que las diferencias obtenidas entre el método de interpolación y la corrección aplicada con el MMC se encuentran entre 1 y $4.5 \mathrm{kt}$.

En la Fig. 4 se puede apreciar como el campo de viento interpolado con el vecino natural es capaz de representar bien el comportamiento de las isotacas del campo de viento del WRF-NMM, este método ofrece valores de la fuerza del viento en el área de interés cercanos a los simulados por el modelo WRF-NMM; además, es capaz
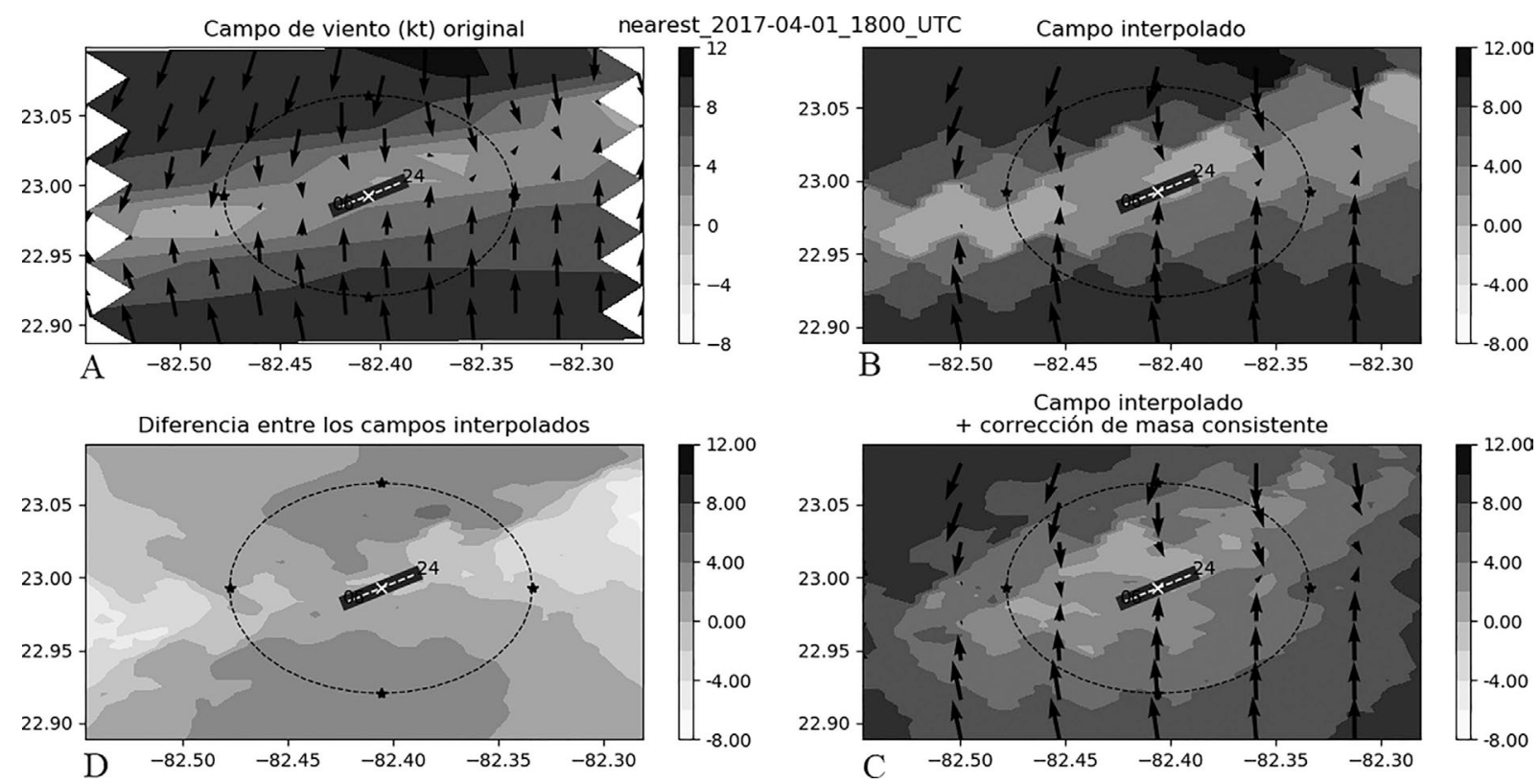

Figura 3 - Dirección y velocidad (kt) del viento a las 1800 UTC del día 01-04-2017: A) Campo de viento obtenido a partir de las salidas numéricas del WRF-NMM. B) Interpolación del campo de viento con el vecino más cercano. C) Corrección aplicada a dichas interpolaciones con el MMC. D) Diferencias entre los campos interpolados. 

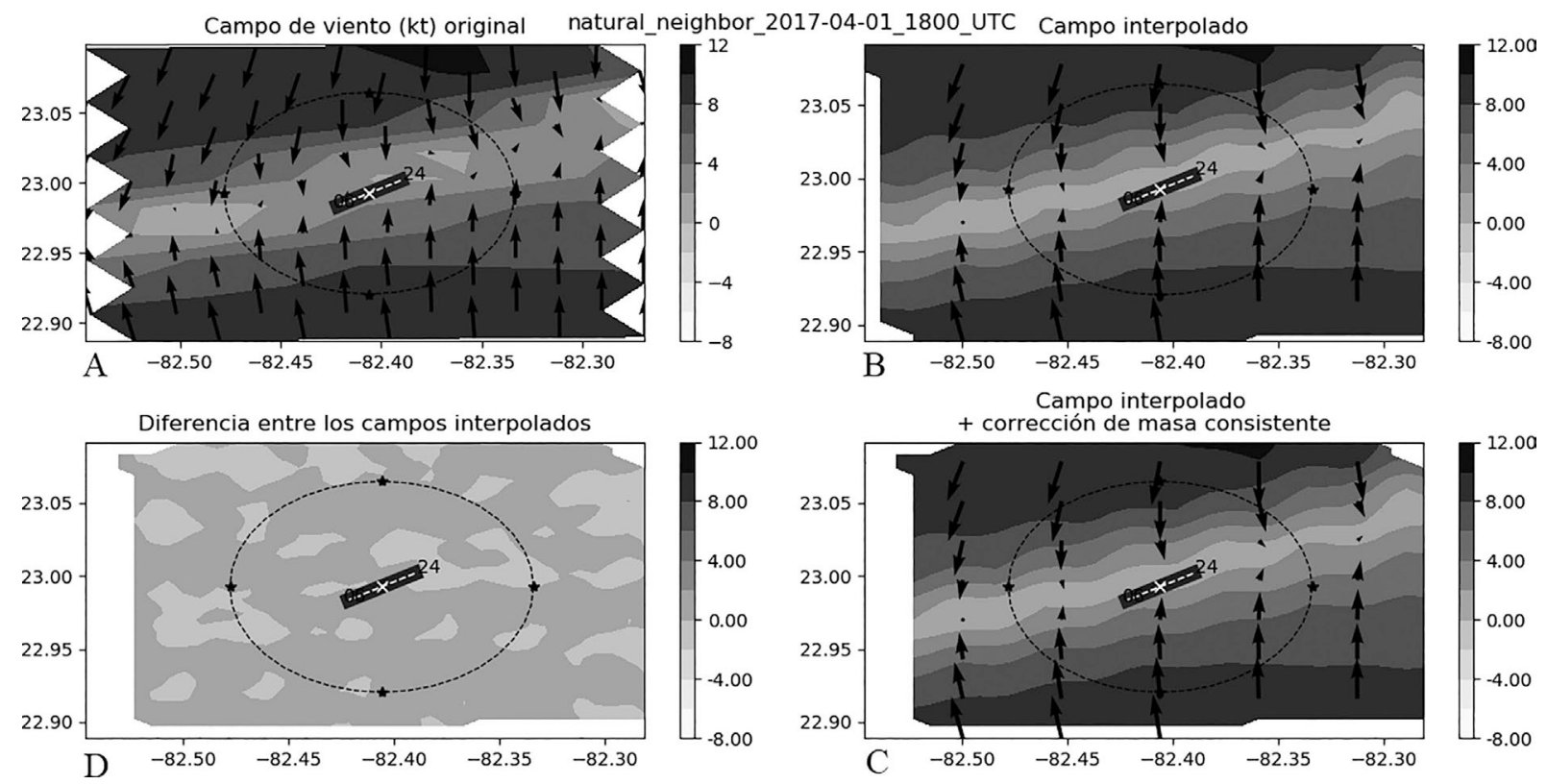

Figura 4 - Dirección y velocidad (kt) del viento a las 1800 UTC del día 01-04-2017: A) Campo de viento obtenido a partir de las salidas numéricas del WRF-NMM. B) Interpolación del campo de viento con el vecino natural. C) Corrección aplicada a dichas interpolaciones con el MMC. D) Diferencias entre los campos interpolados.

de conservar la dirección del viento. También se observa como al aplicarle a dicho método de interpolación una corrección con el MMC los resultados obtenidos son muy similares, siendo mínimas las diferencias obtenidas entre el método de interpolación y la corrección aplicada.

En el caso de la interpolación realizada utilizando el spline cúbico, el método lineal y las RBF los resultados fueron similares a los obtenidos con el vecino más cercano (Fig. 3).

\subsection{Verificación y evaluación de los resultados}

Se alcanzaron resultados similares para todos los pronósticos numéricos del campo de viento realizados para el centro y las cabeceras de la pista del MUHA. A continuación, se muestran los obtenidos para la RWY 24 a partir de las 0600 UTC con un plazo de validez de 24 horas; ya que se tuvo en cuenta el período que necesita el modelo para adaptarse a la topografía y suavizar los campos. Se seleccionó la RWY 24; debido a que en este punto se obtuvieron los mejores resultados, porque es el punto más cercano a un nodo de la malla del modelo.

Para la fuerza del viento los valores simulados fueron mayores a los datos reales encontrándose el BIAS entre 0 y $4 \mathrm{kt}$,; los mayores errores se encontraron en las primeras 6 horas de pronóstico, siendo los errores aproximadamente de $4.5 \mathrm{kt}$ cuando se hace uso de los métodos de interpolación lineal, spline cúbico, RBF y cercanos a los $3.5 \mathrm{kt}$ empleando el vecino más cercano y el vecino natural; en las próximas 9 horas los errores disminuyen siendo cercanos a los $0 \mathrm{kt}$ lo que indica la cercanía de los valores simulados y los reales, en las restantes horas los errores vuelven a aumentar. Para la dirección del viento el comportamiento del BIAS fue similar, oscilando dichos valores entre $-25^{\circ}$ y $5^{\circ}$, subestimando los distintos métodos a los valores reales en la mayoría de las horas de pronóstico (Fig. 5).

En la Fig. 6, se observa que el error absoluto medio osciló entre 2 y $3.8 \mathrm{kt}$, los que disminuyeron en $0.5 \mathrm{kt}$ al aplicarle la corrección con el modelo de Masa Consistente. En el caso de la dirección del viento el MAE presentó valores inferiores a los $50^{\circ}$.

El índice de habilidad del pronóstico (Fig. 7) del campo de viento presentó valores entre 0.1 y 0.7 para todos los métodos de interpolación para la fuerza y dirección del viento, presentando los mejores resultados el método de interpolación del vecino natural.

La correlación de la velocidad del viento presentó valores en el rango de 0.5 a 0.8 , lo que indica que la relación lineal entre los valores simulados y los reales es buena. Para la dirección del viento la correlación osciló entre 0.2 y 0.65 , indicando que la relación lineal es poca en las primeras horas y buena en las últimas 12 horas. El coeficiente de ajuste osciló entre 0.6 y 0.85 , lo que indica cuan cerca se encuentran los valores simulados de los reales. El índice de dispersión presentó valores entre 0.05 y 0.3 , por lo que los resultados obtenidos se encuentran poco dispersos de los valores reales.

\subsection{Comparación entre las herramientas numéricas para el pronóstico del campo de viento}

En las Fig. 8 (A, B, C, D) se muestran los resultados obtenidos para el pronóstico numérico de la fuerza $(\mathrm{kt})$ y 

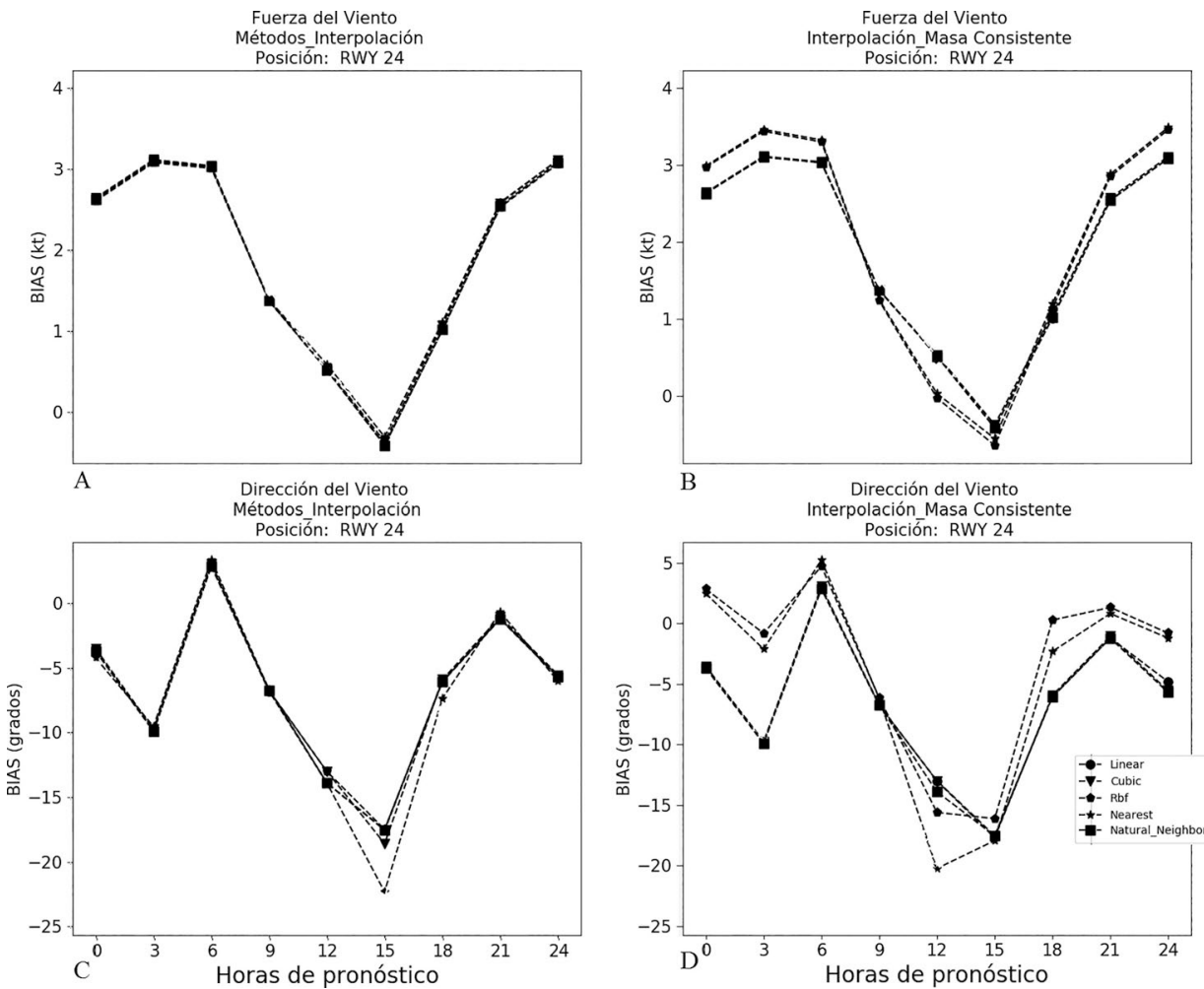

Figura 5 - Evaluación realizada con el BIAS en la RWY 24 para la: A) Fuerza del viento interpolada (kt). B) Fuerza del viento interpolada más la corrección con el MMC (kt). C) Dirección del viento interpolada (grados) D) Dirección del viento interpolada más la corrección con el MMC (grados).
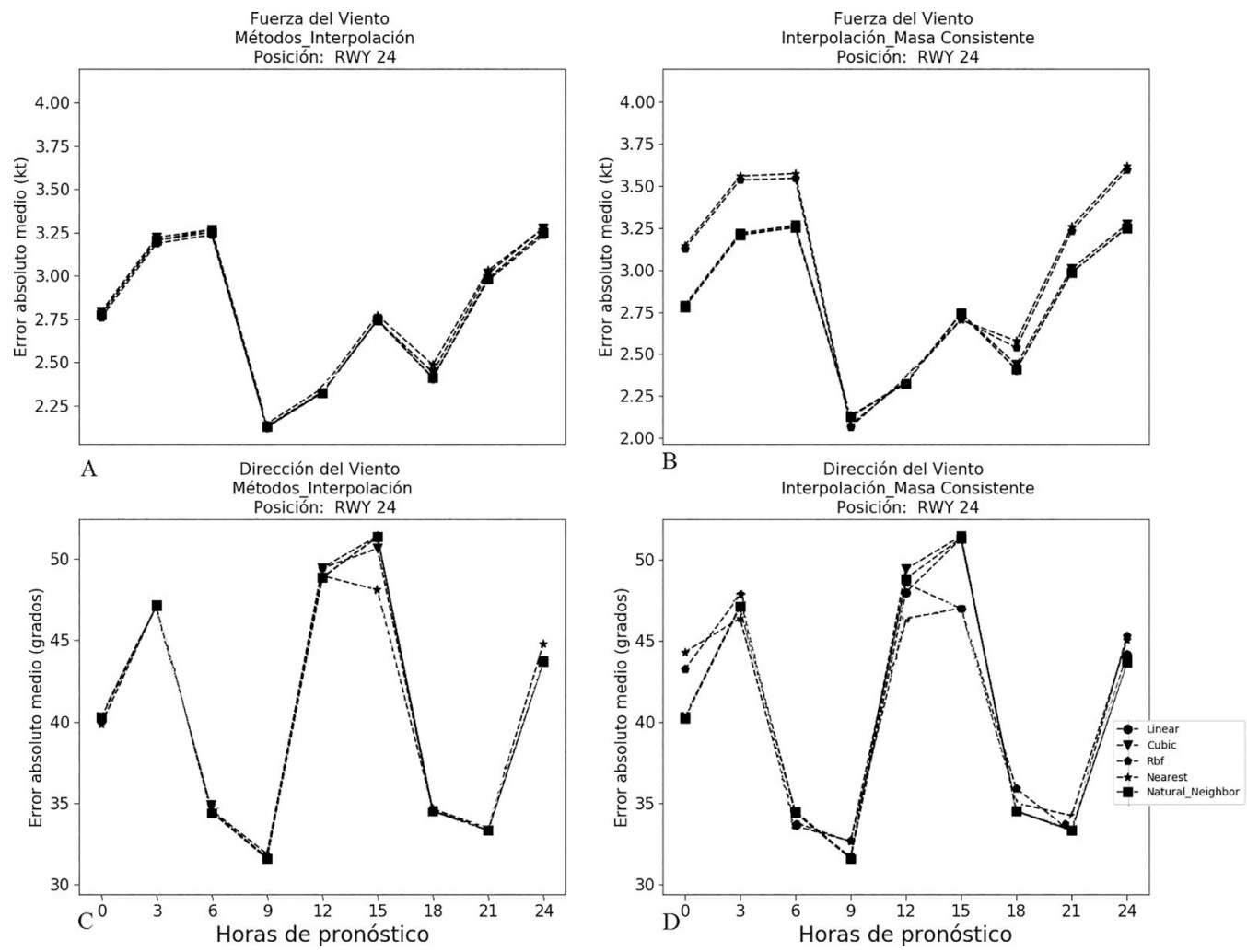

Figura 6 - Evaluación realizada con el MAE en la RWY 24 para la: A) Fuerza del viento interpolada (kt). B) Fuerza del viento interpolada más la corrección con el MMC (kt). C) Dirección del viento interpolada (grados) D) Dirección del viento interpolada más la corrección con el MMC (grados). 

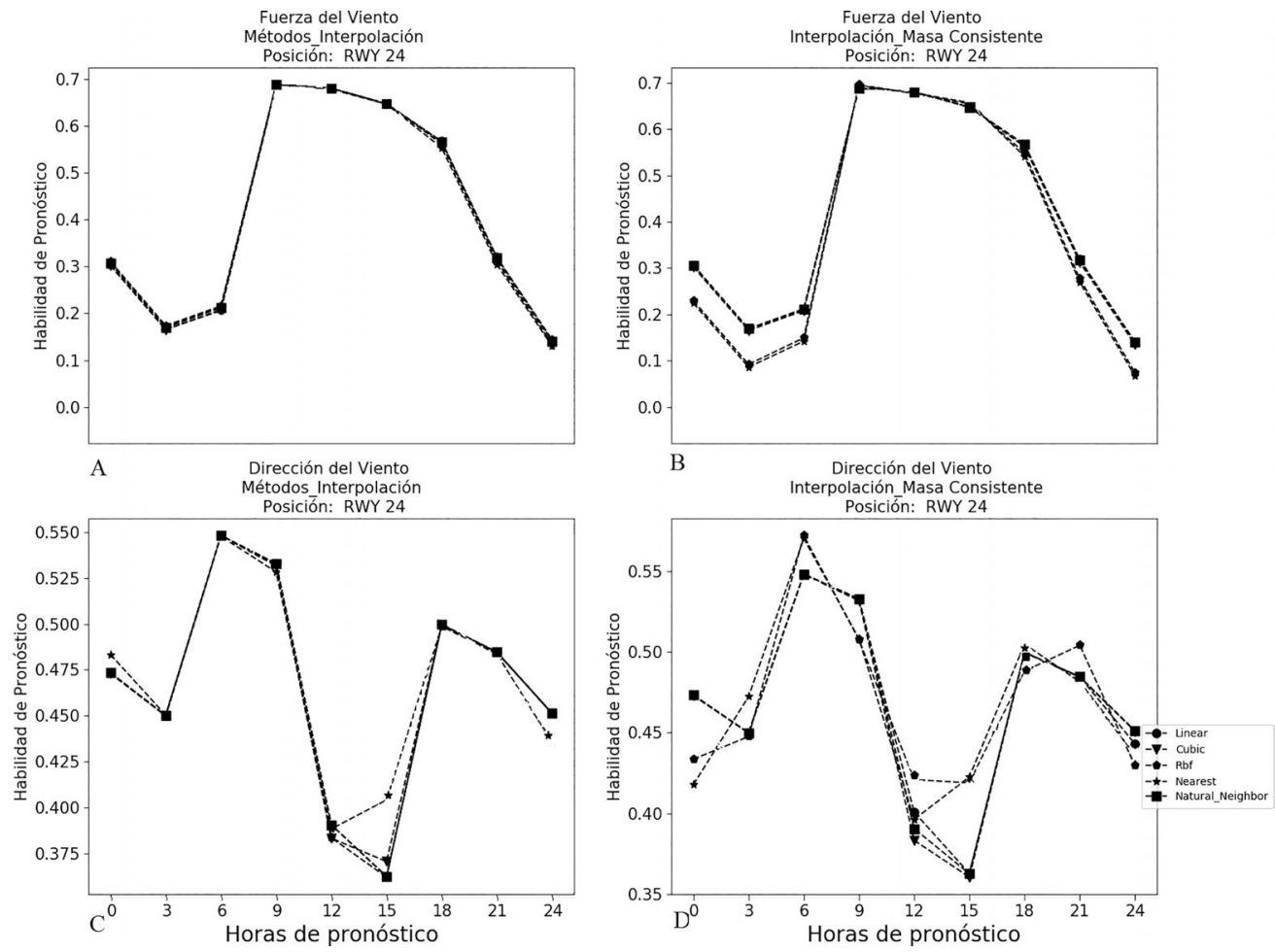

Figura 7 - Evaluación realizada con el SS en la RWY 24 para la: A) Fuerza del viento interpolada (kt). B) Fuerza del viento interpolada más la corrección con el MMC (kt). C) Dirección del viento interpolada (grados) D) Dirección del viento interpolada más la corrección con el MMC (grados).
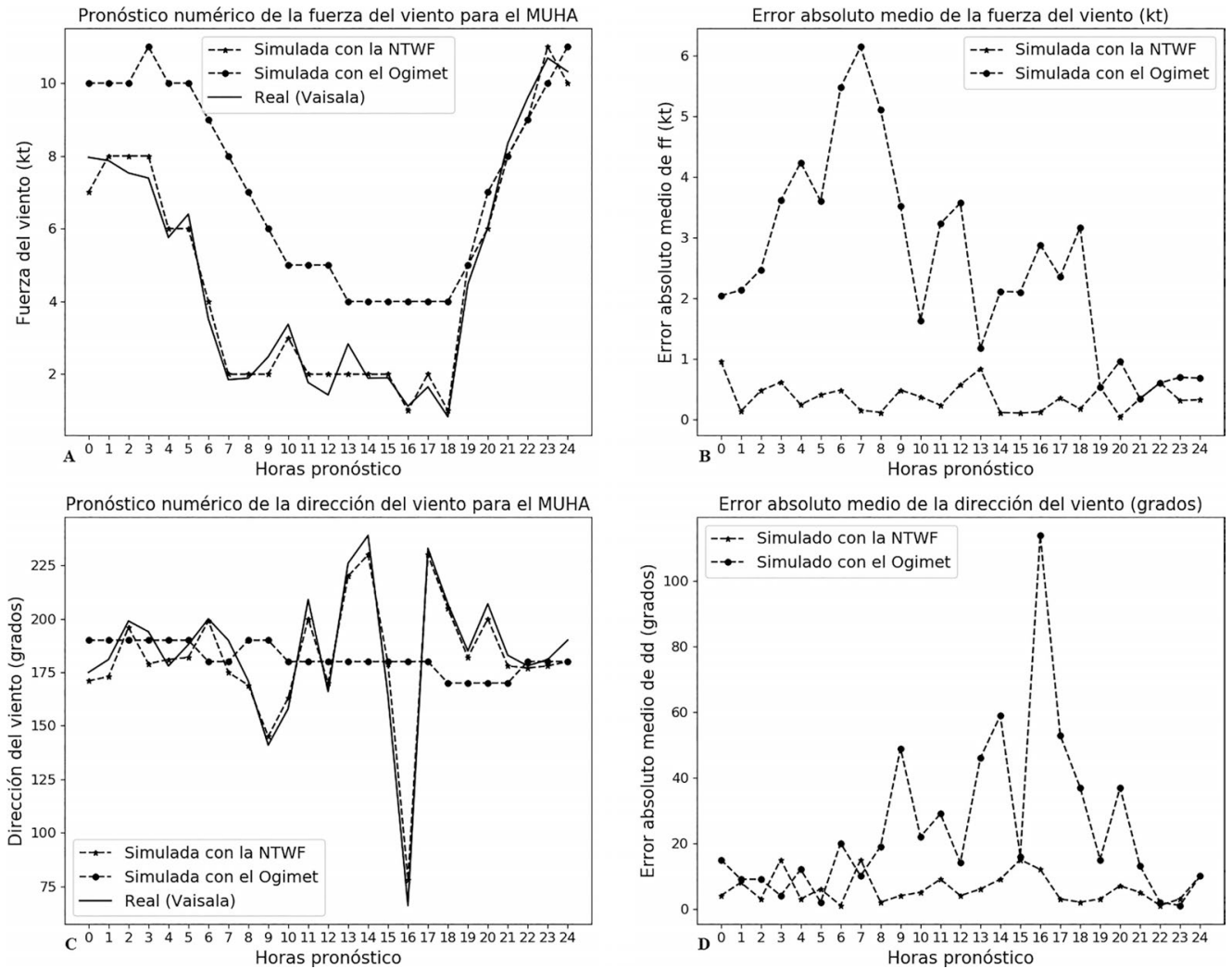

Figura 8 - A) Pronóstico numérico de la fuerza del viento (kt). B) Error absoluto medio de la fuerza del viento (kt). C) Pronóstico numérico de la dirección del viento (grados). D) Error absoluto medio de la dirección del viento (grados). 
dirección del viento (grados) con la herramienta desarrollada Numerical Tools Weather Forecast (NTWF, por sus siglas en inglés) y con el Ogimet, desde el 10-05-2019 hasta el 16-05-2019 para la RWY 24. En las mismas se puede apreciar que la NTWF es capaz de representar mejor el comportamiento de la fuerza y dirección del viento real (Väisälä) que el Ogimet. El error absoluto medio de la NTWF oscila entre 0 y $1 \mathrm{kt}$, siendo $0 \mathrm{kt}$ en varias horas pronósticos, lo que indica la exactitud de los valores simulados con respecto a los valores reales; empleando el Ogimet el error absoluto medio presentó valores entre 1 y 6 kt. Para la dirección del viento los errores medios oscilaron entre 0 y 20 grados para la NTWF, mientras que para el Ogimet presentaron valores entre 0 y 40 grados y un pico de 120 grados entre las 15 y 17 horas pronósticos.

En la Tabla 2 se muestran los resultados de la efectividad (por ciento que representa el dato simulado del valor real) de los pronósticos numéricos del campo de viento para las $0000,0600,1200$ y 1800 UTC, realizados con la NTWF y el Ogimet desde el 10-05-2019 hasta el 16-052019 para la RWY 24. Se puede apreciar que para todas las horas pronósticos la NTWF presentó una superioridad con respecto al Ogimet en la determinación del campo del viento.

Los resultados obtenidos con la NTWF presentan una superioridad de 30 grados para la dirección y de $5 \mathrm{kt}$ para la velocidad del viento con respecto a los interpolados directamente de las salidas numéricas del modelo WRF-NMM.

\subsection{Pronósticos numéricos obtenidos}

Se obtuvieron los pronósticos numéricos del campo de viento para el centro y las cabeceras de la pista del MUHA para las 0000, 0600, 1200 y 1800 UTC con un período de validez de 24 horas. En la Fig. 9 se observa el pronóstico numérico obtenido para la RWY 24, válido desde las 0000 UTC del 10-04-2019 hasta las 2300 UTC del 10-04-2019.

Estos pronósticos numéricos del campo de viento se encuentran operativos en la Oficina Principal y de Vigilancia Meteorológica (OPVM) de la Empresa Cubana de Navegación Aérea (ECNA S.A), en la Oficina de Aeródromo de la Habana (AISMET HAV) perteneciente también a la ECNA S.A y en el Departamento de Meteorología del Instituto Superior de Tecnologías y Ciencias Aplicadas (INSTEC). Para su visualización se confeccionó un sitio WEB disponible en la página del INSTEC. Este producto se actualiza en tiempo real automáticamente, garantizando el trabajo de los meteorólogos aeronáuticos de forma operativa.

Tabela 2 - Efectividad de los pronósticos numéricos de la fuerza y dirección del viento.

\begin{tabular}{|c|c|c|c|c|c|c|c|c|}
\hline \multirow[t]{3}{*}{ Fecha } & \multicolumn{8}{|c|}{ Efectividad de los pronósticos numéricos de la fuerza del viento (\%) } \\
\hline & \multicolumn{4}{|c|}{ NTWF } & \multicolumn{4}{|c|}{ OGIMET } \\
\hline & 00 UTC & 06 UTC & 12 UTC & 18 UTC & $00 \mathrm{UTC}$ & 06 UTC & $12 \mathrm{UTC}$ & $18 \mathrm{UTC}$ \\
\hline 2019-05-10 & 89 & 87 & 88 & 89 & 30 & 19 & 27 & 33 \\
\hline 2019-05-11 & 90 & 92 & 89 & 92 & 28 & 58 & 32 & 21 \\
\hline 2019-05-12 & 95 & 96 & 93 & 94 & 20 & 25 & 31 & 35 \\
\hline 2019-05-13 & 89 & 87 & 90 & 92 & 30 & 16 & 29 & 39 \\
\hline 2019-05-14 & 96 & 93 & 89 & 88 & 32 & 39 & 64 & 45 \\
\hline 2019-05-15 & 97 & 97 & 88 & 90 & 45 & 42 & 30 & 15 \\
\hline 2019-05-16 & 88 & 90 & 91 & 90 & 50 & 32 & 41 & 32 \\
\hline Promedio & 92 & 92 & 90 & 91 & 34 & 33 & 36 & 36 \\
\hline \multirow[t]{3}{*}{ Fecha } & \multicolumn{8}{|c|}{ Efectividad de los pronósticos numéricos de la dirección del viento (\%) } \\
\hline & \multicolumn{4}{|c|}{ NTWF } & \multicolumn{4}{|c|}{ OGIMET } \\
\hline & 00 UTC & 06 UTC & 12 UTC & 18 UTC & $00 \mathrm{UTC}$ & 06 UTC & $12 \mathrm{UTC}$ & 18 UTC \\
\hline 2019-05-10 & 91 & 90 & 90 & 90 & 82 & 82 & 68 & 67 \\
\hline 2019-05-11 & 85 & 95 & 94 & 94 & 65 & 58 & 53 & 56 \\
\hline 2019-05-12 & 95 & 94 & 95 & 96 & 55 & 61 & 83 & 83 \\
\hline 2019-05-13 & 97 & 97 & 97 & 93 & 83 & 66 & 69 & 69 \\
\hline 2019-05-14 & 94 & 92 & 91 & 90 & 37 & 38 & 33 & 18 \\
\hline 2019-05-15 & 89 & 88 & 84 & 83 & 26 & 20 & 55 & 50 \\
\hline 2019-05-16 & 86 & 86 & 86 & 86 & 30 & 36 & 46 & 30 \\
\hline Promedio & 91 & 92 & 91 & 90 & 54 & 52 & 58 & 53 \\
\hline
\end{tabular}


Pronóstico de campo de viento para MUHA-RWY 24

Válido desde 2019-10-04 a las 00 UTC hasta 2019-10-04 a las 23 UTC
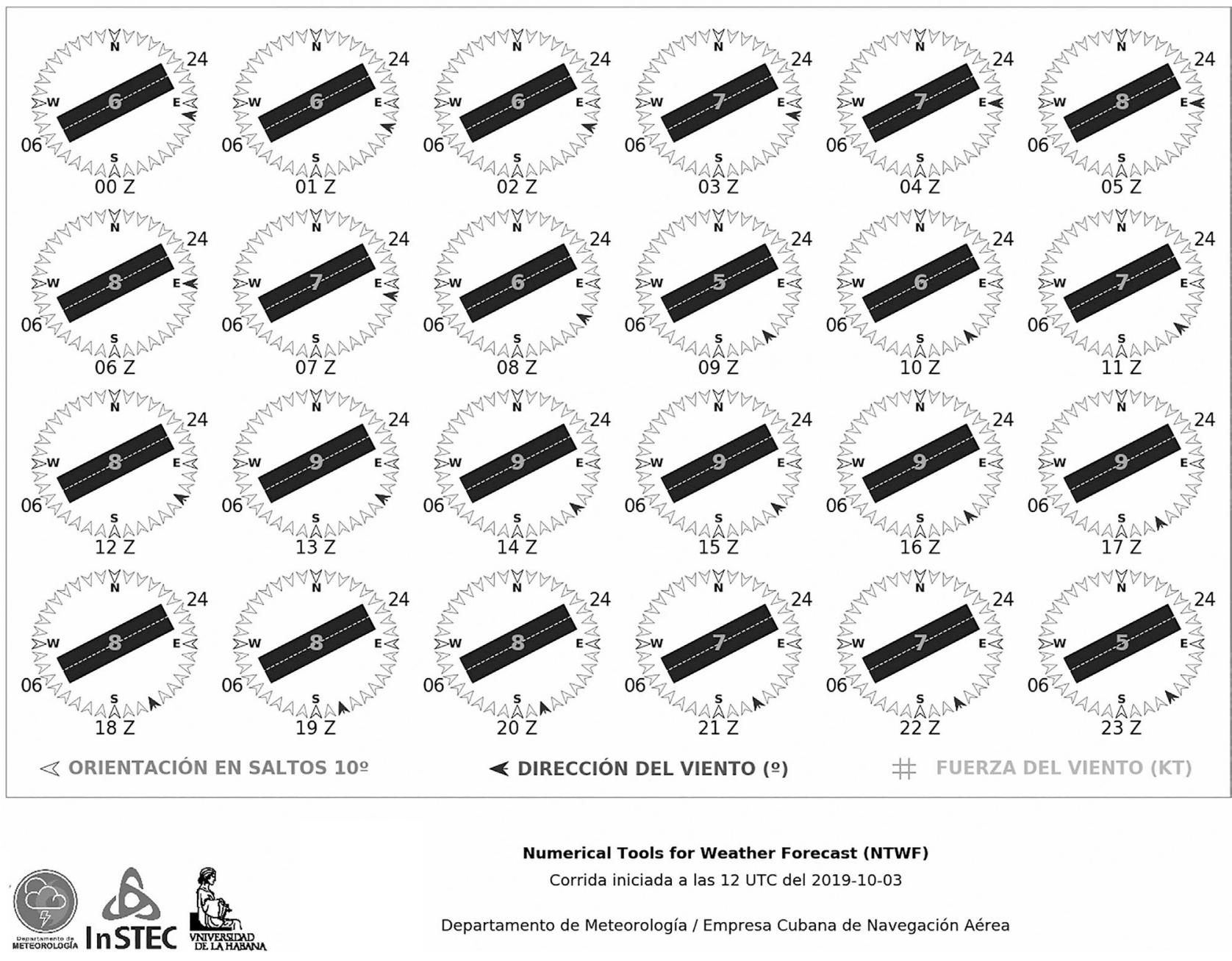

Numerical Tools for Weather Forecast (NTWF)

Corrida iniciada a las 12 UTC del 2019-10-03

Departamento de Meteorología / Empresa Cubana de Navegación Aérea

Figura 9 - Pronóstico numérico del campo de viento para el MUHA

\section{Conclusiones}

Se obtuvo el pronóstico numérico del campo de viento para la RWY 06, RWY 24 y el MID del Aeropuerto Internacional "José Martí" de La Habana correspondientes a las 0000, 0600, 1200, 1800 UTC con una efectividad del 91\% para el pronóstico numérico del campo de viento.

En el período de estudio para todas las horas pronosticadas la corrección con el modelo de Masa Consistente aplicada al vecino natural ofrece los mejores resultados con una sobrestimación máxima de $3 \mathrm{kt}$ para la fuerza y de $20^{\circ}$ para la dirección del viento.

Los resultados alcanzados para la fuerza y dirección del viento se consideran satisfactorios; ya que la Regulación Aeronáutica Cubana (IACC, 2013) exige como límite $10 \mathrm{kt}$ (en un rango $\pm 5 \mathrm{kt}$ ) para la fuerza y $60^{\circ}$ (en un rango $\pm 30^{\circ}$ ) para la dirección del viento.
La herramienta desarrollada para el pronóstico numérico del campo de viento del Aeropuerto Internacional "José Martí" de La Habana presentó una habilidad superior al Ogimet.

\section{Referencias}

ÁLVAREZ, M.; GUERRA, A.; LAU, R. Libro de Matemática Numérica. La Habana: Poligráfica Félix Varela, p. 227270, 2009.

BALLESTER, G.; GARCÍA, J.M. Guía para usuarios de Ogimet. Recuperado el 21 de septiembre de 2018, de www. ogimet.com.

DE ARMAS, A.M.; CREMATA, L.; PÉREZ, L. Caracterización de la dirección y fuerza del viento en el Aeropuerto Internacional "Abel Santamaría” en el período 2011-2015. Revista Cubana de Meteorología, v. 25, n. 1, p. 2, 2019. 
ENTENZA, L. Comportamiento de los fenómenos meteorológicos peligrosos para la aviación civil en el Aeropuerto Internacional "José Martí”. In: V Congreso Cubano de Meteorología, Ciudad de la Habana, 2009.

HERNÁNDEZ, G.M.; LEÓN, E.M. Métodos de Interpolación Multivariable. Tesis en opción al título de Licenciatura en Matemática. Universidad del Salvador, Facultad de Ciencias Naturales y Matemáticas, p. 28-30, 2016.

IACC. Regulación Aeronáutica Cubana (RAC-3). La Habana: Instituto de Aeronáutica Civil de Cuba, $6^{\text {ta }}$ ed, cap IV, $4^{\text {ta }}$ sección, art 24: p. 5, 2013

IACC. Publicación de Información Aeronáutica (AIP). La Habana: Instituto de Aeronáutica Civil de Cuba, p. 40, 2014.

IACC. Manual de Meteorología Aeronáutica (MAC). La Habana: Instituto de Aeronáutica Civil de Cuba. Anexo H: Utilización de la información meteorológica para la planificación de las operaciones previas al vuelo por los explotadores y las tripulaciones de vuelo, $6^{\text {ta }}$ ed, p. H2, Junio 2017.

JÁCOME, D.A. Modelo de Masa Consistente para Aproximar Campos de Viento 3D. Tesis en opción al título de Máster en Matemáticas Aplicadas e Industriales. Universidad Autónoma Metropolitana Iztapalapa, México. División de Ciencias Básicas e Ingeniería, p. 38-49, 2014

JANJIC, Z.; BLACK, T.; PYLE, M.; FERRIER, B.; CHUANG, H. et al. NMM version 3 modelling system user's guide. Developmental Testbed Center / National Centers for Enviromental Prediction, p. 1-213, 2014.

JIMÉNEZ, A. Modelo de Masa Consistente para la Simulación Numérica del Campo del Viento. Tesis en opción al título de Licenciatura en Meteorología. Instituto Superior de Tecnologías y Ciencias Aplicadas, La Habana, Cuba, p. 17-44, 2012.

JOLliFE, I.T.; STEPHENSON, D.B. Forecast Verification. A Practitioner's Guide in Atmospheric Science. Hoboken: John Wiley y Sons Ltd, p. 27, 99-106, 2003.

LAZZARO, D.; MONTEFUSCO, L.B. Radial basis functions for the multivariate interpolation of large scattered data sets. Journal of Computational and Applied Mathematics, v. 140, p. 521-524, 2002.

MÉNDEZ, A.; CALVO, J. Evaluación de un modelo 3D de elementos finitos y Masa Consistente para la predicción de viento a muy alta resolución. In: XXXIV Jornadas Científicas de la Asociación Meteorológica Española, Teruel, p. 1-5, 2016.

MONTERO, G.; MONTENEGRO, R.; ESCOBAR, J.M.; RODRÍGUEZ, E. Simulación Numérica De Campos De Vientos: Implementación De Un Modelo Tridimensional De Masa Consistente. Las Palmas, Instituto Universitario de Sistemas Inteligentes y Aplicaciones Numéricas en Ingeniería, p. 1-8, 2005.

PÉREZ-BELLO, A.; MITRANI-ARENAL, I.; DÍAZ-RODRÍGUEZ, O.; WETTRE, C.; HOLE, L.R. A numerical pre- diction system combining ocean, waves and atmosphere models in the Inter-American Seas and Cuba. Revista Cubana de Meteorología, v. 25, n. 1, p. 109-120, 2019.

PÉREZ, L. Características Del Viento Cruzado En Las Temporadas Invernales Del Período 2001-2004 En El Aeropuerto Internacional “José Martí”. Tesis presentada en opción al Título de Máster en Meteorología, UDICT, Instituto de Meteorología, pp. 79, 2006.

ROBLES, R. La cizalladura descafeinada. Revista del Aficionado a la Meteorología, v. 8, n. 1, p. 1-4, 2003.

RODRÍGUEZ, E. MONTERO, G.; MONTENEGRO, R.; ESCOBAR, J.M.; RODRÍGUEZ-JIMÉNEZ, E. Uso combinado de modelos de viento a escala local. In: XXI Congreso de Ecuaciones Diferenciales y Aplicaciones, XI Congreso de Matemática Aplicada. Instituto Universitario de Sistemas Inteligentes y Aplicaciones Numéricas en Ingeniería. Universidad de Las Palmas, Gran Canaria, p. 1-8, 2009.

RATTO, C.F.; FESTA, R.; ROMEO, C.; FRUMENTO, O.A.; GALLUZZI, M. Mass consistent models for wind fields over complex terrain: The estate of the art. Environmrntal Software, v. 9, n. 4, p. 247-268, 1994.

SIBSON, R. Una Breve Descripción De La Interpolación Del Vecino Natural. Interpolación De Datos Multivariados. Chichester: John Wiley, $5^{\text {ta }}$ ed, p. 21-36, 1981.

SIERRA, M.; FERRER, A.L.; HERNÁNDEZ, R.; GONZÁLEZ, Y.; CRUZ, R.C. et al. Sistema Automático De Predicción A Mesoescala De Cuatro Ciclos Diarios. Informe De Resultado. La Habana: Instituto de Meteorología, p. 32-47, 2015.

STOLZ, W.; ROJAS, J.; CHINCHILLA, R. Climatología aeronáutica (2000-2010) en el Aeropuerto Internacional Tobías Bolaños Palma, Costa Rica. San Jose: Instituto Meteorológico Nacional, p. 10-11, 2012.

TORRES, S. Fenómenos Reductores De La Visibilidad Y EI Viento Cruzado En El Aeropuerto Internacional "José Martí". Tesis en opción al título de Licenciatura en Meteorología. Instituto Superior de Tecnologías y Ciencias Aplicadas, La Habana, Cuba, p. 53-56, 2011.

\section{Recursos de Internet}

Ogimet, www.ogimet.com.

NCEP, https://nomads.ncep.noaa.gov/cgi-bin/filter_gfs_0p50.pl. INSTEC, https://www.instec.cu/model/airport_products.php.

1Distancia mínima normada: a $150 \mathrm{~m}$ de la zona de toma de contacto de las cabeceras y el centro de la pista.

License information: This is an open-access article distributed under the terms of the Creative Commons Attribution License (type CC-BY), which permits unrestricted use, distribution and reproduction in any medium, provided the original article is properly cited. 